\title{
Phases, many-body entropy measures, and coherence of interacting bosons in optical lattices
}

\author{
R. Roy, ${ }_{1}$ A. Gammal, ${ }^{2}$ M. C. Tsatsos, ${ }^{3}$ B. Chatterjee, ${ }^{4}$ B. Chakrabarti, ${ }^{1,2}$ and A. U. J. Lode ${ }^{5,6,7}$ \\ ${ }^{1}$ Department of Physics, Presidency University, 86/1 College Street, Kolkata 700 073, India \\ ${ }^{2}$ Instituto de Física, Universidade de São Paulo, CEP 05580-090, São Paulo, Brazil \\ ${ }^{3}$ Instituto de Física de São Carlos, Universidade de São Paulo, CP 369,13560-970, São Carlos, SP, Brazil \\ ${ }^{4}$ Department of Physics, Indian Institute of Technology Kanpur 208016, India \\ ${ }^{5}$ Department of Physics, University of Basel, CH-4056 Basel, Switzerland \\ ${ }^{6}$ Wolfgang Pauli Institute c/o Faculty of Mathematics, University of Vienna, Oskar-Morgenstern Platz 1, A-1090 Vienna, Austria \\ ${ }^{7}$ Vienna Center for Quantum Science and Technology, Atominstitut, TU Wien, Stadionallee 2, A-1020 Vienna, Austria
}

(Received 7 December 2017; revised manuscript received 13 March 2018; published 24 April 2018)

\begin{abstract}
Already a few bosons with contact interparticle interactions in small optical lattices feature a variety of quantum phases: superfluid, Mott-insulator, and fermionized Tonks gases can be probed in such systems. To detect these phases-pivotal for both experiment and theory-as well as their many-body properties we analyze several distinct measures for the one-body and many-body Shannon information entropies. We exemplify the connection of these entropies with spatial correlations in the many-body state by contrasting them to the Glauber normalized correlation functions. To obtain the ground state for lattices with commensurate filling (i.e., an integer number of particles per site) for the full range of repulsive interparticle interactions we utilize the multiconfigurational time-dependent Hartree method for bosons (MCTDHB) in order to solve the many-boson Schrödinger equation. We demonstrate that all emergent phases-the superfluid, the Mott insulator, and the fermionized gas can be characterized equivalently by many-body entropy measures and by Glauber's normalized correlation functions. In sharp contrast, single-particle entropy cannot capture these phases.
\end{abstract}

DOI: 10.1103/PhysRevA.97.043625

\section{INTRODUCTION}

Ultracold atoms provide a testing ground for many-body physics [1]. Utilizing Feshbach resonance management, the interatomic interaction can be tuned to any desired value [2]. This tunability allows the production of weakly or strongly correlated Bose-Einstein condensates (BECs). A variety of different geometries and topologies is realizable by suitably manipulating the magneto-optical trapping potentials [3]. For instance, interacting bosons in optical lattices, that is, a spatially periodic potential, have been shown to exhibit a quantum phase transition from a superfluid state (SF) to a Mott insulator (MI) [4-6].

One-dimensional optical lattices loaded with strongly interacting bosons [7] have been shown to be experimentally more challenging than their three-dimensional counterparts: Quantum fluctuations are not negligible [8], trigger correlations $[9,10]$ and are the focus of the present paper.

The zero-temperature SF to MI transition is commonly described by the Bose-Hubbard model [11]. For weak interparticle interactions, a Bose gas in an optical lattice of moderate depth is in the superfluid phase: The many-body state features long-range coherence. This SF phase persists, as long as the interatomic interaction is small compared to the tunneling coupling-i.e., the parameter of the BoseHubbard Hamiltonian that determines the lattice depth and hence tunneling between neighboring sites. The SF can be characterized as a state in which all particles are delocalized between all sites of the lattice. When the repulsive interaction is large compared to the tunneling coupling, each site is filled up with an identical number of bosons and the coherence between different sites is completely lost: The many-body state enters the Mott-insulating phase.

Even though the Bose-Hubbard model can aptly describe the above SF to MI transition in lattices, its regime of validity is restricted to the case where site-localized Wannier states are an appropriate basis set [12]. It has been demonstrated that a general quantum many-body description, valid at all interaction strengths, is necessary for the regime beyond the Bose-Hubbard model $[13,14]$. One such theory is the multiconfigurational time-dependent Hartree (MCTDH) method [15]. In Ref. [16] MCTDH was applied to small lattices and it was found that correlations depend both on the commensurability and the strength of the interparticle interactions. In the present paper, we solve the full many-body Schrödinger equation at a high level of accuracy by using the multiconfigurational timedependent Hartree method for bosons (MCTDHB) [17-19]. We note that MCTDHB has been applied to the dynamics of ultracold bosons in multiwell traps successfully [20-25]. In the following, we will use the open source implementation of MCTDHB in the MCTDH-X software [26-29]. The motivation for our present work is to investigate the pathway from the superfluid through the Mott insulating to the fermionized phase [30] and explain it in terms of production of many-body information entropy.

Entropies of quantum systems were long ago introduced [31-40] and used as a measure of the degree of order of a given quantum state [41-46]. Entropy measures for many-body states were shown to saturate in time to the values given by estimates of Gaussian orthogonal ensembles (GOE) of random matrices 
for the time evolution triggered by an interaction quench [47]. This situation, when different entropy measures take on the GOE values, is referred to as statistical relaxation and was shown to also reflect itself in the correlation functions of the many-body state [47].

Below, we analyze this connection of many-body entropies and correlation functions for the quantum phases of many-body states in an optical lattice, commensurately filled with bosons that interact with a repulsive contact interaction. We characterize the many-body state by calculating the produced entropy and the spatial correlations as a function of the interparticle interaction strength. We demonstrate that the production of different types of entropies-defined below - can be used to identify the phase of the system.

We quantify the coherence properties of all the emergent phases in one-dimensional lattices - superfluid, Mott insulator, and fermionized phase-by computing Glauber's normalized first- and second-order correlation functions. We find that the classification of phases using correlation functions agrees with the classification of phases using many-body entropy measures. The fundamental relation between many-body entropies and coherence properties in ultracold bosonic atoms, found in Refs. [47,48] in the time evolution following a quench, is extended to the present case of stationary states in lattices.

The paper is structured as follows. In Sec. II we expose the Hamiltonian and introduce the many-body theory and our quantities of interest: Glauber correlation functions and manybody entropy measures. Section III deals with the results of our analysis of ground states for various interaction parameters and filling factors. We conclude our work in Sec. IV.

\section{THEORY}

\section{A. Hamiltonian}

Consider a system of $N$ bosons interacting with a contact interparticle interaction potential in one spatial dimension. In dimensionless units, such a system is governed by the following Hamiltonian:

$$
H=\sum_{i=1}^{N}\left(-\frac{1}{2} \frac{\partial^{2}}{\partial x_{i}^{2}}+V\left(x_{i}\right)\right)+\lambda \sum_{i<j}^{N} \delta\left(x_{i}-x_{j}\right) .
$$

Here, $V(x)=V_{0} \sin ^{2}(k x)$ is the lattice potential, where $k=\frac{\pi}{d}$; $V_{0}$ is the depth and $d$ is the periodicity of the lattice. The strength of the two-body interactions $\lambda$ can be experimentally tuned almost at will in quasi-one-dimensional systems by manipulating the strength of the transversal confinement [49]. In the remainder of the paper, we set a depth of $V_{0}=12.0$ in order to showcase all phases and in particular the fermionization limit without the necessity to simulate an enormously large interparticle interaction strength. Further, we set our grid to range from $x_{\min }=-4.7124 \approx-\frac{3}{2} \pi$ to $x_{\max }=4.7124 \approx \frac{3}{2} \pi$ such that $d=3$ wells are considered. The depth $V_{0}$ is chosen such that it allows superfluidity, for appropriately chosen boson number $N$ and interaction strength $\lambda$. We find the stationary solutions of the many-body Schrödinger equation with periodic boundary conditions and obtain the observables defined in Sec. IIC as a function of the strength of the interparticle interactions $\lambda$ and the number of atoms per lattice site.

\section{B. MCTDHB}

In the multiconfigurational time-dependent Hartree for bosons (MCTDHB) approach, the wave function of the interacting $N$-boson problem is expanded over a set of permanents. Permanents are symmetrized bosonic states of $N$ particles in $M$ single-particle states. Each permanent can be constructed by acting products of $N$ boson creation operators $b_{k}^{\dagger}(k=1, \ldots, M)$ onto the vacuum $|\mathrm{vac}\rangle$ :

$$
\begin{gathered}
|\Psi(t)\rangle=\sum_{\vec{n}} C_{\vec{n}}(t)|\vec{n} ; t\rangle ; \\
|\vec{n} ; t\rangle=\left|n_{1}, n_{2}, \ldots n_{M} ; t\right\rangle=\prod_{i=1}^{M}\left[\frac{\left(b_{i}^{\dagger}(t)\right)^{n_{i}}}{\sqrt{n_{i} !}}\right]|\mathrm{vac}\rangle .
\end{gathered}
$$

Here, each operator $b_{k}^{\dagger}(t)$ creates a boson occupying the timedependent single-particle state (orbital) $\phi_{k}(x, t)$. The number of possible configurations of $N$ bosons in $M$ orbitals is equal to $\left(\begin{array}{c}N+M-1 \\ N\end{array}\right)$ and defines the number of complex-valued coefficients $C_{\vec{n}}(t)$ in Eq. (2). A formal variational treatment with the above ansatz leads to the MCTDHB equations of motion [17-19]. The solution of the latter yields the time evolution of the coefficients $C_{\vec{n}}(t)$ and orbitals $\phi_{k}(x, t)$ that built up our solution: an approximation to the solution of the timedependent Schrödinger equation at a desired (arbitrary) degree of accuracy Refs. [50-52]. We remark here that the formulation of MCTDHB does at no point require the permanents $|\vec{n} ; t\rangle$ to be built up. The unfavorable scaling of the computation of the permanent with the number of particles $N$ is circumvented because MCTDHB relies on second quantization.

In this work, we find the eigenstates of the Schrödinger equation by propagating the MCTDHB equations in imaginary time using the MCTDH-X package [26-28]. In the limit of infinitely many orbitals, $M \rightarrow \infty$, the set of permanents $\left|n_{1}, n_{2}, \ldots n_{M} ; t\right\rangle$ in Eq. (3) spans the complete $N$-particle Fock space and MCTDHB becomes exact [50-52].

\section{Quantities of interest}

We now introduce the quantities that we use to characterize the solutions of the Schrödinger equation.

(a) Entropies. The Shannon information entropies of the one-body density in coordinate space $\rho^{(1)}(x)=$ $\left\langle\Psi\left|\hat{\Psi}^{\dagger}(x) \hat{\Psi}(x)\right| \Psi\right\rangle$ is given by

$$
S_{x}(t)=-\int d x \rho^{(1)}(x, t) \ln \left[\rho^{(1)}(x, t)\right] .
$$

Analogously, for the momentum density, $\rho^{(1)}(k)=$ $\left\langle\Psi\left|\hat{\Psi}^{\dagger}(k) \hat{\Psi}(k)\right| \Psi\right\rangle$ we have

$$
S_{k}(t)=-\int d k \rho^{(1)}(k, t) \ln \left[\rho^{(1)}(k, t)\right] .
$$

The SIEs in Eqs. (4) and (5) are a measure of the delocalization of the corresponding distributions $\rho^{(1)}(x)$ and $\rho^{(1)}(k)$. References [41-43] establish a universal relation between entropy and the number of interacting particles for diverse systems like atoms, nuclei, and atomic clusters. Since the distributions in Eqs. (4) and (5) are related to the one-body density, they are insensitive to correlations that may be present in the state $|\Psi\rangle[29,48,53-64]$. One can, 
however, formulate an SIE using the two-body density distributions $\rho^{(2)}\left(x_{1}, x_{2}\right)=\left\langle\Psi\left|\hat{\Psi}^{\dagger}\left(x_{1}\right) \hat{\Psi}^{\dagger}\left(x_{2}\right) \hat{\Psi}\left(x_{1}\right) \hat{\Psi}\left(x_{2}\right)\right| \Psi\right\rangle$ and $\quad \rho^{(2)}\left(k_{1}, k_{2}\right)=\left\langle\Psi\left|\hat{\Psi}^{\dagger}\left(k_{1}\right) \hat{\Psi}^{\dagger}\left(k_{2}\right) \hat{\Psi}\left(k_{1}\right) \hat{\Psi}\left(k_{2}\right)\right| \Psi\right\rangle \quad$ (see Ref. [65]):

$$
S_{\rho-x}(t)=-\int d x_{1} d x_{2} \rho^{(2)}\left(x_{1}, x_{2} ; t\right) \ln \left[\rho^{(2)}\left(x_{1}, x_{2} ; t\right)\right],
$$

and

$$
S_{\rho-k}(t)=-\int d k_{1} d k_{2} \rho^{(2)}\left(k_{1}, k_{2} ; t\right) \ln \left[\rho^{(2)}\left(k_{1}, k_{2} ; t\right)\right] .
$$

Here $\rho^{(2)}\left(x_{1}, x_{2}\right)\left[\rho^{(2)}\left(k_{1}, k_{2} ; t\right)\right]$ is the diagonal part of the twobody reduced density matrix in position [momentum] space. Since the SIEs in Eqs. (6) and (7) are computed from twobody quantities, they are measures sensitive to correlations in the many-body state $|\Psi\rangle$. By comparing the SIE based on the one-body density [Eqs. (4) and (5)] with the SIE based on the two-body density [Eqs. (6) and (7)] the presence of correlations in the state $|\Psi\rangle$ can be inferred.

Since the state we consider is expanded in the MCTDHB theory as $|\Psi\rangle=\sum_{\vec{n}} C_{\vec{n}}(t)|\vec{n} ; t\rangle$, we can define an alternative SIE using the coefficients $C_{\vec{n}}$ that characterize the distribution of the state $|\Psi\rangle$ in the underlying Fock space [cf. Eq. (3)]:

$$
S_{c}(t)=-\sum_{\vec{n}}\left|C_{\vec{n}}(t)\right|^{2} \ln \left[\left|C_{\vec{n}}(t)\right|^{2}\right] .
$$

We term this entropy coefficient Shannon information entropy (C-SIE), or simply coefficient entropy. A mean-field state is a single-configuration state, i.e., only a single coefficient contributes in Eq. (3). For such a state $S_{c}(t)=0$ holds at all $t$. Coefficient entropy $S_{c}$ thus cannot be produced in a mean-field theory. When the state $|\Psi\rangle$ spreads across several configurations $|\vec{n} ; t\rangle$, several expansion coefficients contribute [cf. Eq. (3)] and the coefficient entropy $S_{c}$ gradually increases. In the limiting case, when the complete $N$-body Fock space is populated by the state $|\Psi\rangle$, all coefficients are equally large and $S_{c}$ saturates to its maximal value.

Last, one can define an SIE measure related to the emergence of fragmentation $[37,47,48,53]$, i.e., the emergence of multiple significant eigenvalues $n_{i} ; i=1, \ldots, M$ of the reduced one-body density matrix $\rho^{(1)}$ of the state $|\Psi\rangle$ [66-68]. These eigenvalues are also referred to as natural occupations and thus we term the following measure of entropy the occupation Shannon information entropy (O-SIE):

$$
S_{n}=-\sum_{i}^{M} n_{i} \ln n_{i}
$$

We remark that this definition of the entropy in the eigenvalues of the reduced one-body density matrix has earlier been developed and studied for fermionic systems [31-36].

In the case of bosons, for a state described by a single-orbital mean-field theory, the reduced density matrix is characterized by only a single eigenvalue and hence $S_{n}=0$ holds. For single-configuration states with multiple contributing orbitals as well as multiconfigurational states, there may be several occupation numbers and hence $S_{n} \neq 0$. For the condensed gas only a single occupation dominates and the occupation entropy is zero. For an increase in interaction strength, the O-SIE gradually increases and saturates only for a maximally fragmented state. (b) Correlation functions. The normalized $p$ th order correlation function is defined by

$$
g^{(p)}\left(x_{1}^{\prime}, \ldots, x_{p}^{\prime}, x_{1}, \ldots, x_{p}\right)=\frac{\rho^{(p)}\left(x_{1}, \ldots, x_{p} \mid x_{1}^{\prime}, \ldots, x_{p}^{\prime}\right)}{\sqrt{\prod_{i=1}^{p} \rho^{(1)}\left(x_{i} \mid x_{i}\right) \rho^{(1)}\left(x_{i}^{\prime} \mid x_{i}^{\prime}\right)}},
$$

and is the key quantity to define spatial $p$ th order coherence. Here, $\rho^{(p)}\left(x_{1}, \ldots, x_{p} \mid x_{1}^{\prime}, \ldots, x_{p}^{\prime} ; t\right)$ is the $p$ th order reduced density matrix of the state $|\Psi\rangle$ [69]. In the case of $\left|g^{(p)}\left(x_{1} \ldots, x_{p}, x_{1} \ldots, x_{p} ; t\right)\right|>1(<1)$, the detection probabilities of $p$ particles at positions $x_{1}, \ldots, x_{p}$ are referred to as (anti)correlated. Recent progress in experiments in quantum gases has been remarkable and the measurement of higher-order correlation functions is now possible [70-74]. In particular, in Ref. [74] it has been explicitly shown how a many-body system is characterized via its higher-order correlations.

\section{RESULTS}

In this section, we display our numerical results for the SIE measures and spatial correlations in the eigenstates of the Hamiltonian of Eq. (1) as a function of the strength of repulsive interactions, $\lambda$. We choose three different sets of system parameters representative for the three distinct phases that the system may be in. Results for the superfluid phase, the Mott insulating phase, and the fermionized phase are discussed in the following Secs. III A, III B, and III C, respectively.

We define the filling factor as the ratio of the number of atoms $N$ and the number of lattice sites $W: v=\frac{N}{W}$. For the entire manuscript we focus on commensurate filling factors $v=1,2$, and 7. We keep the lattice depth $V_{0}=12.0$ and the number of lattice sites $W=3$ fixed. We thus change the number of particles to change the filling factor and the interaction strength is gradually tuned to cover all the emergent phases. The transitions between the emergent phases are discriminated using information entropies and correlation functions.

We choose to present the results in the order of increasing complexity of the found many-body state: $v=7,1,2$ in Secs. III A, III B, and III C, respectively. Indeed, we find that the $v=7$ case is captured within a mean-field theory for the range of the interparticle interaction strengths which we investigate. The $v=7$ and $v=1$ cases can qualitatively be described within the Bose-Hubbard model as there is no structure that forms within sites. Notably, the fermionization that emerges for the $v=2$ case at stronger interparticle interactions is a manybody phase that neither of the aforementioned approaches can describe.

\section{A. Commensurate filling factor $v=7$ : Superfluid state}

Here, we characterize the superfluid phase using natural occupations, normalized correlation functions, and the Shannon information entropy measures that we have introduced. In Fig. 1(a), we plot the occupations as a function of the interaction strength $\lambda$. For relatively weak interactions, only the first natural orbital is occupied and the population of the second and third orbital remain below $10 \%$ for all values of $\lambda<0.01$.

We use the criterion of Penrose and Onsager [66] to identify Bose condensation by the eigenvalues of the reduced density matrix: The system is a condensate if its reduced one-body density matrix has a single macroscopic eigenvalue. From 


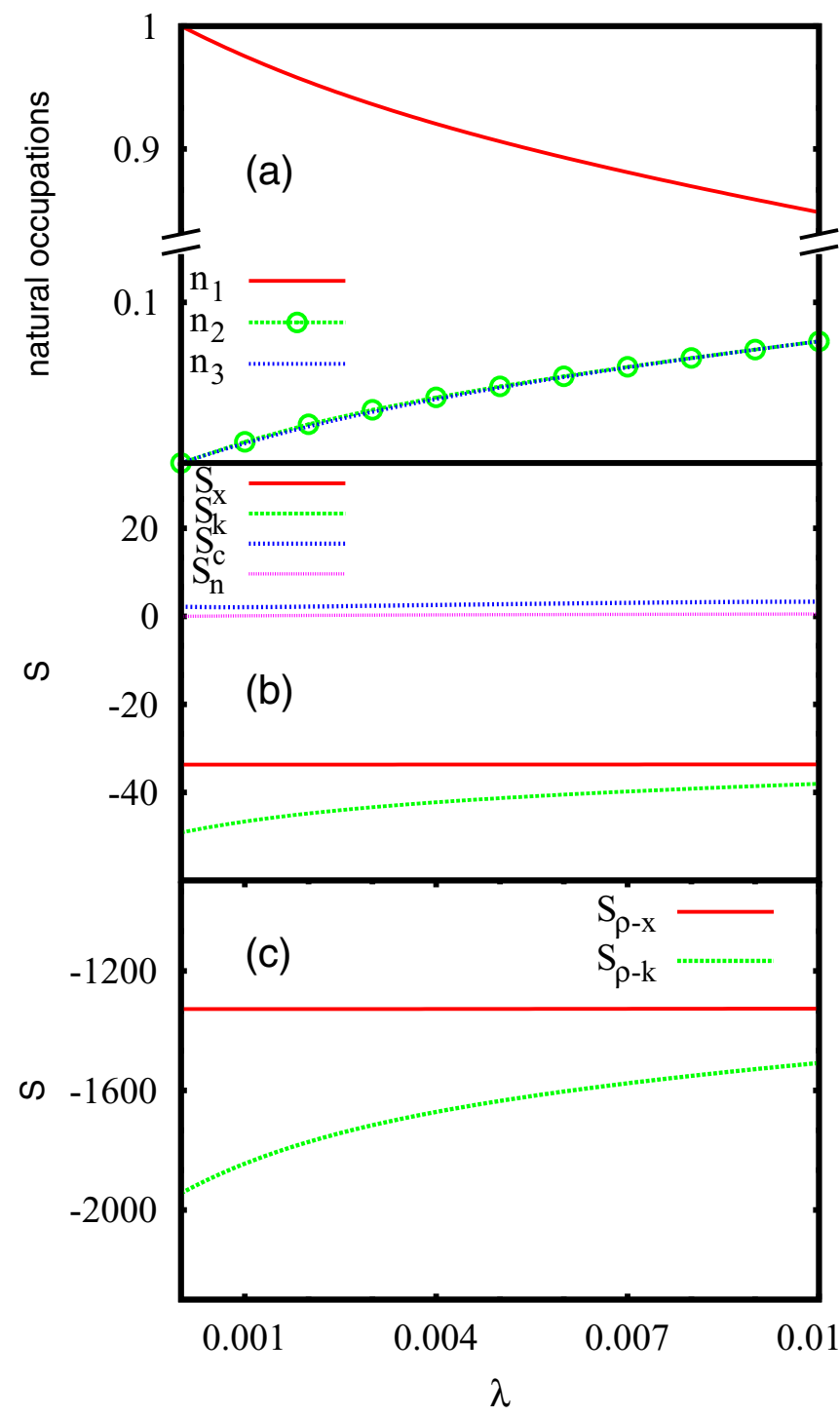

FIG. 1. Entropies and occupations in the superfluid phase. All quantities are shown for $v=7$ and $N=21$ bosons as a function of the interaction strength $\lambda$. (a) Eigenvalues of the reduced density matrix - the ground state is condensed and only a single eigenvalue is macroscopic for the superfluid phase. (b) One-body SIEs $S_{x}$ and $S_{k}$, C-SIE $S_{c}$ and O-SIE $S_{n}$. (c) Two-body SIEs $S_{\rho-x}$ and $S_{\rho-k}$. All quantities are dimensionless. See text for discussion.

Fig. 1(a) it follows that our $v=7$ state can be considered a condensate for all the interaction strengths $\lambda$ considered.

One can, thus, well approximate the many-body wave function with a single-orbital mean-field state $|N, 0, \ldots\rangle$. To achieve convergence in the occupations, i.e., the eigenvalues of the reduced one-body density matrix the interaction strengths that we consider, three orbitals $[M=3$, cf. Eq. (3)] are enough; adding more orbitals does not quantitatively change the manybody state.

We plot the one-body SIE for the density and momentum distributions as a function of the interaction strength $\lambda$ in Fig. 1(b). As $\lambda$ gradually increases the (momentum) density within each well is broadened (narrowed) and consequently the SIE computed from the (momentum) density distribution, $S_{x}$
$\left(S_{k}\right)$ [see Eqs. (4) and (5)] increases (decreases). In Fig. 1(b), we also present the C-SIE $S_{c}$ and O-SIE $S_{n}$. As anticipated, the values of $S_{c}$ and $S_{n}$ are very close to zero, because the condensate is phase coherent and can be well described by a macroscopically occupied single-particle state and the GrossPitaevskii mean-field equation yields a good description.

In Fig. 1(c), we plot the two-body SIE [Eqs. (6) and (7)]. The two-body SIEs behave similarly to the one-body entropy measures. However, the rate of increase in $S_{\rho-x}$ is greater than that in $S_{x}$ and, likewise, the rate of decrease of $S_{\rho-k}$ is greater than that in $S_{k}$. A similar trend was observed in the calculation of one-electron and two-electron entropies in Ref. [65]. The fact that the two-body density matrix canto a good approximation-be written as a product of onebody densities for the coherent superfluid gas is a possible explanation for this behavior.

In Fig. 2(a), we plot the absolute value of the normalized first-order correlation function, $\left|g^{(1)}\left(x^{\prime}, x\right)\right|^{2}$ for $\lambda=0.01$. We see that in the $\left(x, x^{\prime}\right)$ region where the density is localized $\left|g^{(1)}\right|^{2} \approx 1$ holds. We infer that coherence within and between sites is maintained. Figure 2(b) shows the two-body correlation function $g^{(2)}\left(x^{\prime}, x, x^{\prime}, x\right) \equiv g^{(2)}\left(x, x^{\prime}\right)$ for the same parameters as in Fig. 2(a). We find $g^{(2)}\left(x, x^{\prime}\right)$ to be close to unity for all $x^{\prime}, x$. Remarkably, second-order coherence between different wells is perfectly maintained: $g^{(2)}\left(x, x^{\prime}\right) \approx 1$ for the off-diagonal $x^{\prime} \neq x$ with $\left|x^{\prime}-x\right|$ larger than the size of a single well. The diagonal part of the two-body correlation function is slightly depleted, i.e., $g^{(2)}\left(x, x^{\prime}\right) \lesssim 1$ for the diagonal $x^{\prime} \sim x$ if $\left|x^{\prime}-x\right|$ is smaller than the size of a single well. This signifies that antibunching starts to develop for particles within the same site due to the repulsive interactions: Second-order coherence is locally decreased, even though the interaction strength $\lambda$ is relatively small.

\section{B. Commensurate filling factor $v=1$ : transition from condensation to fragmentation}

We now turn our attention to the Mott insulating phase and investigate its many-body physics from the viewpoint of correlation functions, coherence, and Shannon information entropies. To obtain an archetypical Mott insulator for strong interactions, we consider $N=3$ bosons in three wells, i.e., a single atom per site. To quantify the departure of the manybody state $|\Psi\rangle$ from a mean-field state in the superfluid-toMott-insulator transition, we plot the expansion coefficients $\left|C_{n}\right|^{2}$ [Eq. (3)] as a function of the index $n$ of the basis states for two choices for the interaction strength: $\lambda=0.01$ which puts the system into a superfluid phase and $\lambda=10.0$ that renders it a Mott insulator [Figs. 3(a), 3(b)]. In the superfluid phase $(\lambda=0.01)$ the number of significant coefficients is only a small portion of the total number of configurations $N_{\text {conf }}=$ $\left(\begin{array}{c}N+M-1 \\ N\end{array}\right)$. We refer to such a state as localized. We emphasize that our use of the term "localized" refers to many-boson Fock space and not real space; localized states are thus close to a mean-field description where only a single coefficient would contribute. However, as $\lambda$ increases a larger amount of coefficients become significant; we refer to such a state as delocalized [cf. $\lambda=10.0$ in Fig. 3(b)].

In Fig. 3(c), we plot the corresponding occupation of the first, second, and third natural orbital as a function of the 


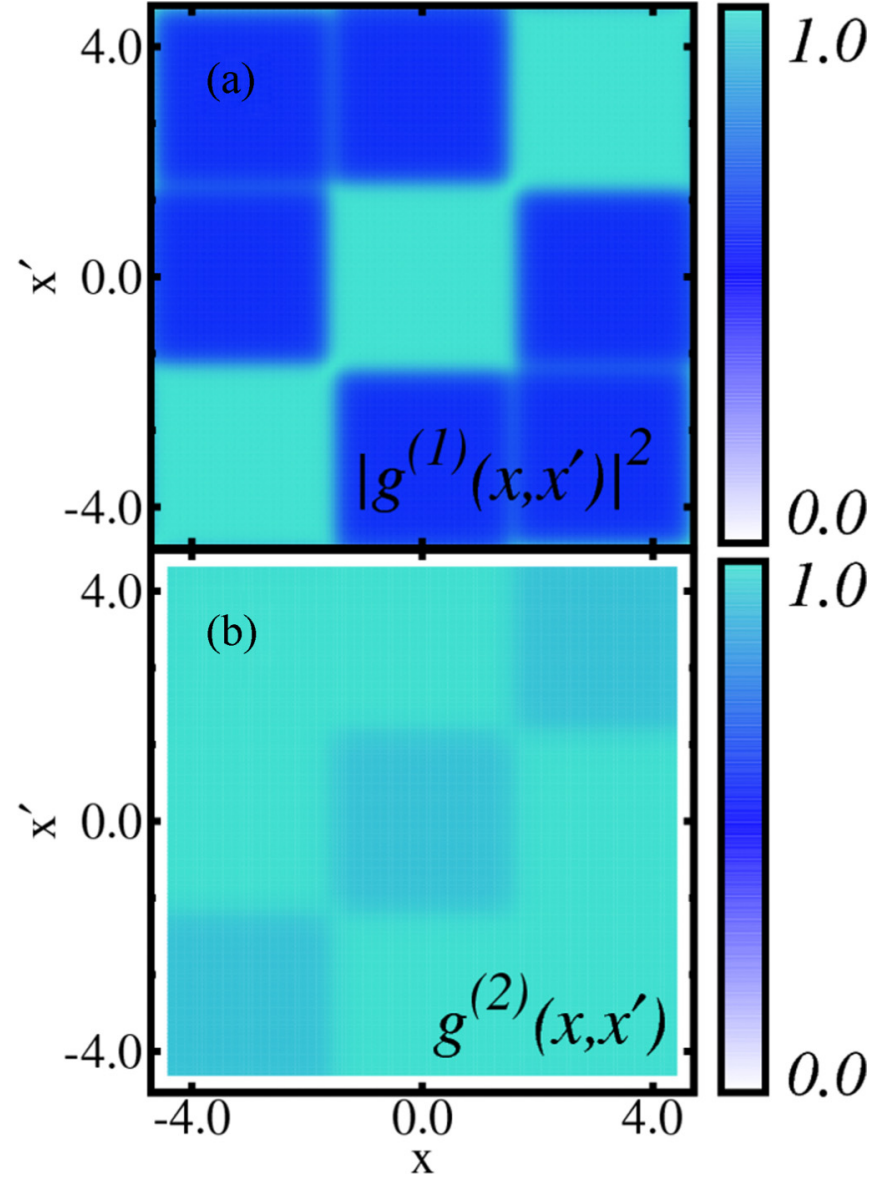

FIG. 2. (a) First- and (b) second-order normalized correlation function for the superfluid phase, i.e., $v=7, N=21$. The interaction strength is $\lambda=0.01$ while the rest of the parameters are as in Fig. 1. (a) Complete first-order coherence is observed within each well; $\left|g^{(1)}\right| \approx 1$ for all $x \approx x^{\prime}$. Significant interwell coherence can be inferred from $\left|g^{(1)}\right| \in[0.5,1]$ for all $x \neq x^{\prime}$ on the off-diagonal. (b) Second-order coherence is retained among distinct wells $g^{(2)}\left(x, x^{\prime}\right) \approx 1$ for all $x \approx x^{\prime}$. The fact that $g^{(2)}$ is maximal among distinct wells but drops within each well indicates that the probability for detecting two particles in different wells is higher than that of finding them in the same well. This behavior is a consequence of the interparticle interactions and it is termed antibunching. All quantities are dimensionless. See text for discussion.

interaction strength. With an increase of $\lambda$, the occupation of the first natural orbital gradually decreases while the occupations of the second and third natural orbitals gradually increase. For large interactions, the first three natural occupations saturate at $33.33 \%$. In our computations with $M=6$ orbitals, all but the first three orbitals had occupations smaller than $10^{-7}$. Threefold fragmentation in a triple well indicates loss of partial coherence of distinct sites and is a signature for the transition to the insulating phase [76]. Thus, across the superfluid-to-Mott-insulator transition the increase of the interaction strength drives the state from a condensed to a fragmented one. Generally, in the Mott-insulating phase there are as many significant eigenvalues of the reduced density matrix as there are lattice sites Ref. [77].

From Fig. 4(a) we see that the above transition cannot be inferred from the density alone. Indeed, the densities as a

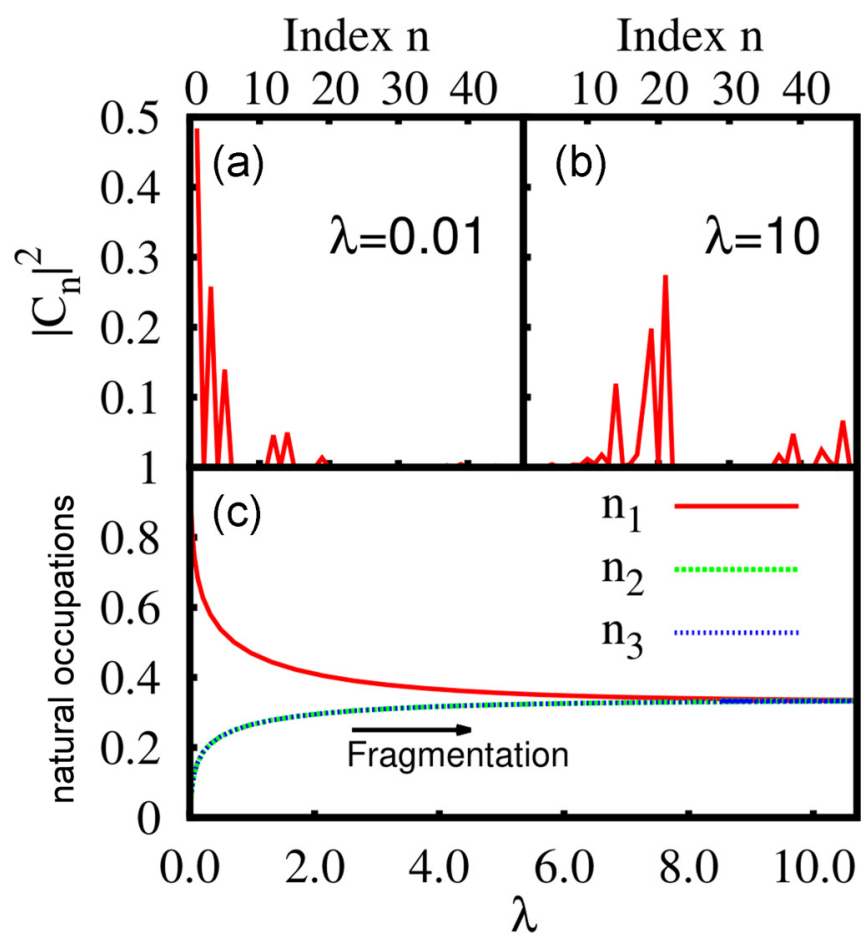

FIG. 3. Localization and delocalization in Fock space in the superfluid-to-Mott-insulator transition at $v=1, N=3$. The distribution of the magnitude of the coefficients $\left|C_{n}\right|^{2}$ as a function of the index $n$ for the superfluid at interaction strength $\lambda=0.01$ in (a) and the Mott insulator at interaction strength $\lambda=10.0$ in (b). The index $n$ is computed from the vector $\vec{n}$ using the mapping described in Ref. [75]. In (a) the $|N, 0, \ldots\rangle$ coefficient and its neighbors dominate while all others are small; the state is localized in many-body Fock space. At (b) the larger interactions force the many-body state to spread over a larger part of the available space and many coefficients are significant; the state is delocalized in many-body Fock space. (c) Populations of the first three natural orbitals. As $\lambda$ increases, the occupation of the first orbital gradually decreases while another two orbitals begin to contribute (green and blue curves). Eventually the state becomes threefold fragmented $\left(n_{1} \approx n_{2} \approx n_{3} \approx 1 / 3\right)$ at $\lambda \gtrsim 6$.0. All quantities are dimensionless.

function of $\lambda$ before and after the transition are identical and many-body measures, such as the entropies, are required to identify the transition. Examining the C-SIE $S_{c}$ and O-SIE $S_{n}$ we observe that for small $\lambda$ both start at zero, as only a single coefficient contributes to the the state, which is a condensed superfluid [Fig. 4(b)]. The behavior of the SIE measures computed from the one- and two-body density are in mutual agreement with the C-SIE and O-SIE measures; compare Figs. 3, 4(b), and 4(c). As $\lambda$ rises, the C-SIE and $\mathrm{O}-\mathrm{SIE}$ measures increase and the many-body state converges to a pure Mott insulator while fragmentation, as well as all SIE measures saturate.

We shall now explore this saturation of entropies using an analogous Gaussian orthogonal ensemble (GOE) [78]. A comparison of MCTDHB results for the C-SIE $S_{c}$ to the entropies in a GOE can hint possible connections to random matrix theory. We stress here that-in contrast to the other entropy measures that we investigate in this work-the value of the C-SIE $S_{c}$ depends on the choice of the many-body basis 


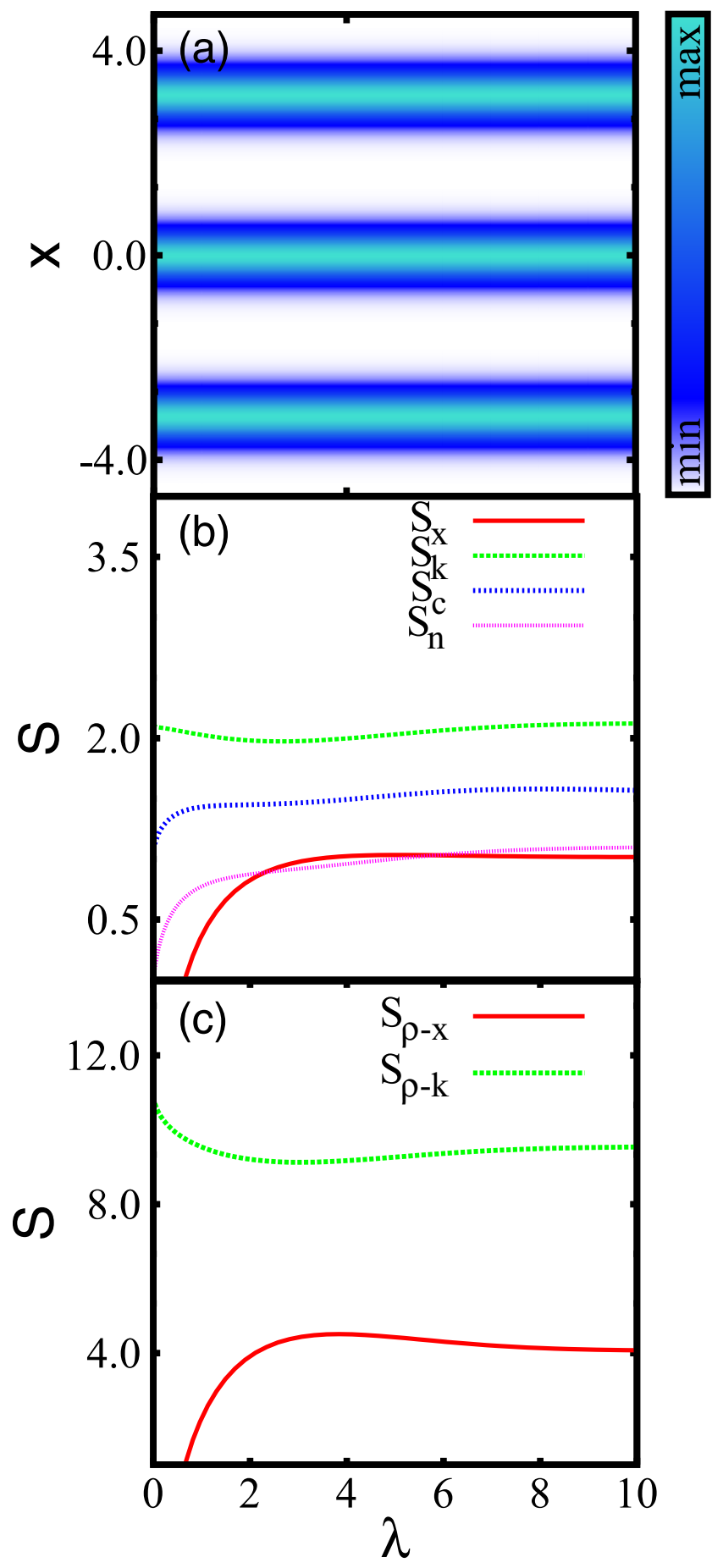

FIG. 4. One-body density and entropies in the superfluid-to-Mottinsulator transition at unit filling $v=1$ and $N=3$ as a function of the interaction strength. (a) The one-body density as a function of interaction strength $\lambda$ shows almost no variation in the transition from a condensed superfluid to a fragmented Mott insulator. (b) Shannon information entropy of the one-body (momentum) density $S_{x}\left(S_{k}\right)$, the occupation entropy $S_{n}$, and the coefficient entropy $S_{c}$ all as a function of $\lambda$. (c) SIE of the reduced two-body (momentum) density matrix $S_{\rho-x}\left(S_{\rho-k}\right)$. For the SIE measures in (b) and (c), the curved part for weak interactions signifies the transition from a condensed superfluid to a fragmented Mott insulator. Within the Mott-insulating phase, for larger interaction strengths $\lambda$, all SIE measures saturate. All quantities are dimensionless; see text for further discussion. used to expand the wave function $|\Psi\rangle$. The wave function given by MCTDHB is invariant under unitary transformations of the single-particle basis set $\left\{\phi_{k}(x, t) ; k=1, \ldots, M\right\}$ that may change the absolute value of the coefficients $\left|C_{\vec{n}}(t)\right|^{2}[17,19]$ and, hence, change the value of $S_{c}$. It would be of particular interest to investigate whether a universal limit with a rigorous connection to random matrix theory exists where the C-SIE $S_{c}$ is entirely independent of the chosen basis.

The GOE of random matrices can be formulated for isolated quantum systems of interacting particles which exhibit time reversal symmetry and rotational invariance [44-46]. The entropies evaluated for the GOE provide estimates for the entropies of many-body systems for the limiting case of maximal disorder. The GOE of random matrices with infinite interaction has entropy $S_{c}^{\mathrm{GOE}}=\ln \left(0.48 D_{c}\right)$, where $D_{c} \times D_{c}$ is the dimension of the considered random matrices.

In the present $v=1$ case, three bosons are distributed in (essentially) three orbitals, $N_{\text {orb }}=3$ [Fig. 3(c)]. Hence, the size of the GOE is $D_{c}=\left(\begin{array}{c}N+N_{\text {orb }}-1 \\ N\end{array}\right)=10$ and thus one obtains $S_{c}^{\mathrm{GOE}}=1.568$. This is in excellent agreement with the value at which our numerical result for the C-SIE $S_{c}$ saturates: 1.552. Despite the mentioned dependence of the coefficients $C_{\vec{n}}$ on the choice of the many-body basis set, the coefficient entropy thus saturates at the value predicted from the GOE for sufficiently large $\lambda$. For the O-SIE $S_{n}$, the dimensionality of the GOE $D_{n}=$ 3 is equal to the number of occupied orbitals in our MCTDHB treatment and $S_{n}^{\mathrm{GOE}}=-\sum_{i=1}^{D_{n}} \frac{1}{D_{n}} \ln \left(\frac{1}{D_{n}}\right)=\ln \left(D_{n}\right)=1.098$. Our numerical result for the saturated value of the O-SIE is identical, $S_{n}=1.098$ [79].

We now analyze the first-order and second-order coherence in the fragmented Mott insulator for $\lambda=10.0$ in Fig. 5. The diagonal of the first-order correlation function shows three completely separated coherent regions where $\left|g^{(1)}\right|^{2} \approx 1$; the coherence of bosons within the same well is maintained while it is lost between distinct wells, since $\left|g^{(1)}\right|^{2} \approx 0$ at the offdiagonal, as expected for the fully localized particles in a Mott insulator. Looking at $g^{(2)}$ we see that coherence is maintained $\left(g^{(2)} \approx 1\right)$ at the off-diagonal but not at the diagonal. The vanishing diagonal part of the normalized two-body correlation function-referred to as the correlation hole [16] - translates to the fact that the probability of finding a double occupation of a single well is practically zero. Second-order coherence between wells is maintained, because the outcome of a twoparticle detection is almost always an uncorrelated detection of two particles in distinct wells.

\section{Commensurate filling factor $v=2$ : from a condensed superfluid via a fragmented Mott insulator to fermionization}

We now consider a system with $v=2$ bosons per site and characterize its phases using the density, normalized correlation functions, and Shannon information entropy measures. In lattices with commensurate filling factor larger than one, i.e., with more than one boson per site, two phase transitions can be observed as a function of the strength of the interparticle interactions $\lambda$ : The superfluid-to-Mott-insulator transition, that is also present if the filling factor is equal to one, emerges for intermediate interaction strengths. For large interaction strengths the Mott-insulator-to-fermionization transition-absent for $v=1-$ now occurs. Since the physics of the superfluid-to- 


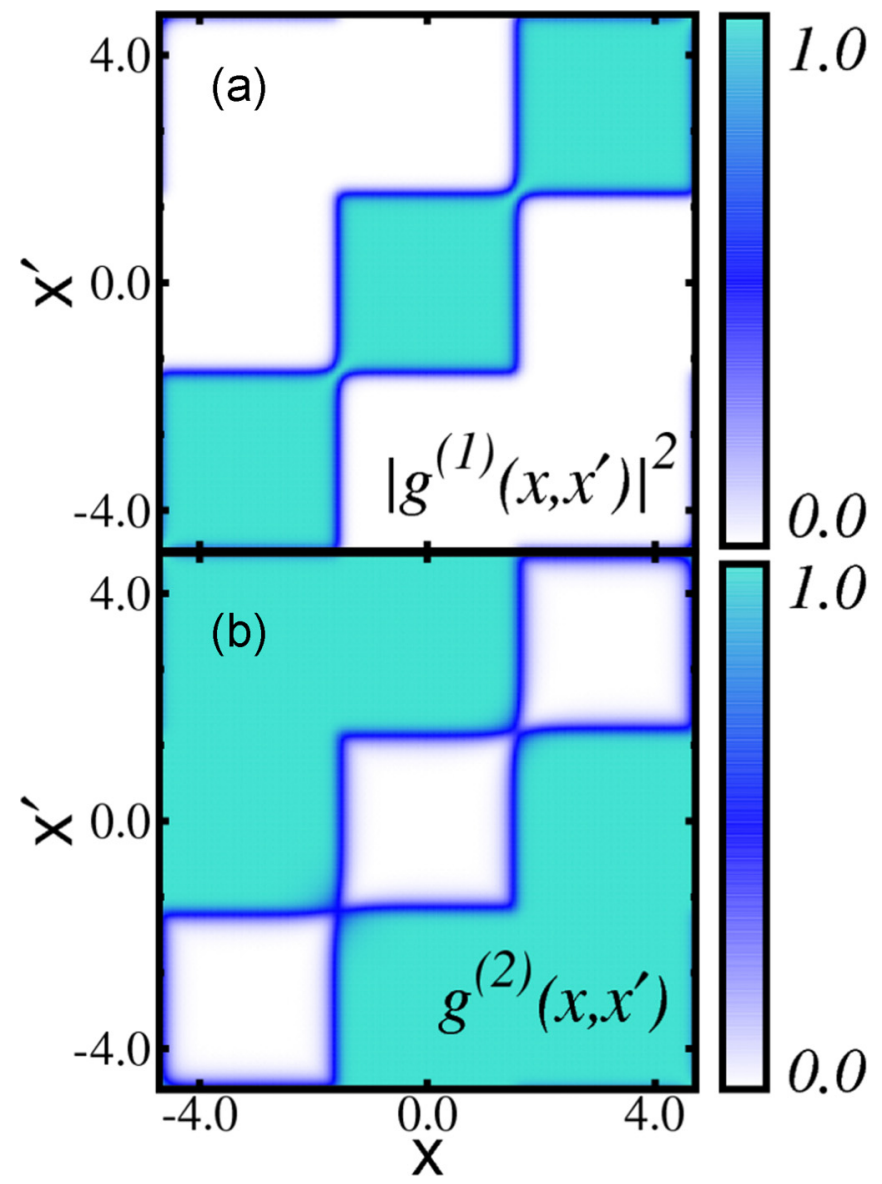

FIG. 5. First-order and second-order coherence of a fragmented Mott insulator at $v=1, N=3$. (a) The normalized first-order correlation function $\left|g^{(1)}\left(x, x^{\prime}\right)\right|^{2}$ for $\lambda=10$ exhibits three separated regions with $\left|g^{(1)}\right|^{2} \approx 1$ along the diagonal and $\left|g^{(1)}\right|^{2} \approx 0$ on the off-diagonal. Thus, the first-order coherence is maintained within wells and lost between them. (b) The normalized second-order correlation function $g^{(2)}\left(x, x^{\prime}\right)$ also for $\lambda=10$. The "correlation hole" is clearly seen and $g^{(2)} \approx 0$ holds for the diagonal $x \approx x^{\prime}$; two particles are thus almost never simultaneously found in the same well. Second-order coherence $g^{(2)} \approx 1$ on the off-diagonal signifies that, in a measurement, two uncorrelated particles will almost surely be detected in distinct wells. The Mott-insulating phase is characterized by independent lattice sites with strongly localized particles. All quantities are dimensionless.

Mott-insulator transition is unchanged, we focus here on the Mott-insulator-to-fermionization transition. The pathway from the condensed superfluid via the fragmented Mott insulator to the fermionization regime depends on the shape of the trapping potential [30]. The optical lattice with filling factor two is the generic system for this transition to be studied.

Similarly to Sec. III B, we see that the system transitions to MI at $\lambda \approx 10$. The transition cannot be seen in the one-body density, since the latter remains unchanged across the SF-to-MI transition as $\lambda$ increases [see Figs. 6(a) and 4(a)]. When the interaction strength $\lambda$ is sufficiently large $(\lambda \gtrsim 200)$, the Mott-insulator-to-fermionization transition takes place and the number of maxima in the one-body density becomes equal to the number of bosons of the system; see Fig. 6(a). In this case the bosonic density resembles a fermionic one where Pauli exclusion forbids the spatial overlap of the particles. The



FIG. 6. One-body density and entropy measures for the transition from the Mott insulator to fermionization. All panels are for $v=$ $2, N=6$ and are plotted against the interaction strength $\lambda$. (a) Plot of the one-body density; the transition to fermionization is visible in the formation of a two-hump intrawell structure - for large $\lambda$, the number of peaks in the density is equal to the number of particles $N$. The dashed contour line $\rho(x)=0.22$ is drawn to visualize the two maxima of each well. (b) SIE computed from the one-body (momentum) density $S_{x}\left(S_{k}\right)$, the coefficients entropy $S_{c}$, and the occupation entropy $S_{n}$. (c) SIE computed from the two-body (momentum) density, $S_{\rho-x}$ $\left(S_{\rho-k}\right)$. Compared to the system with a single boson per well, the SIE measures saturate only for comparatively large interaction strengths [compare (b) and (c) to Figs. 4(b) and 4(c), respectively]. All quantities are dimensionless; see text for further discussion. 


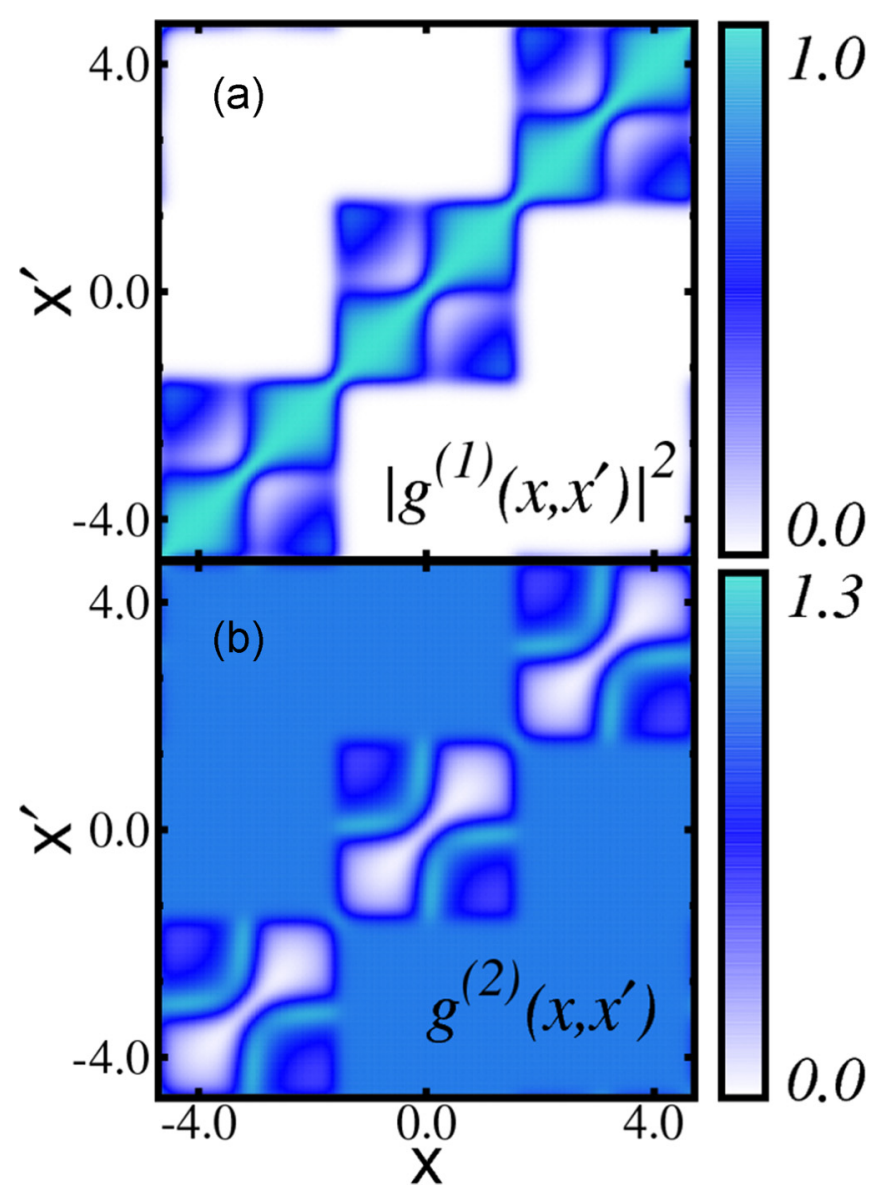

FIG. 7. First- and second-order coherence for $N=6$ fermionized bosons and $v=2$. Both panels show normalized correlation functions for an interaction strength $\lambda=200$. (a) The normalized first-order correlation function $\left|g^{(1)}\left(x, x^{\prime}\right)\right|^{2}$. The coherence between wells is completely lost, i.e., $\left|g^{(1)}\right|^{2} \approx 0$ for the off-diagonal where $x$ and $x^{\prime}$ are positions in distinct wells. The fermionization of the system is manifest in the loss of first-order coherence also within each of the wells [cf. Fig. 5(a)]. (b) The normalized second-order correlation function $g^{(2)}\left(x, x^{\prime}\right)$ has a fully developed correlation hole, $\left|g^{(2)}\right| \approx 0$ on the diagonal. No bunching or antibunching is seen between particles in distinct wells, i.e., $g^{(2)} \approx 1$ on the off-diagonal. Fermionization is manifested in the emergent structures as long as $x$ and $x^{\prime}$ are positions in the same well [cf. Fig. 5(b)]. All quantities shown are dimensionless; see text for further discussion.

transition to fermionization is thus manifest in the formation of intrawell structure in the density. Such intrawell structures cannot be appropriately described by the single-band Hubbard model. When a complete set of Wannier states is considered in each site, i.e., a multiband Hubbard model, an intractably large number of bands quickly becomes necessary [14]. Thus, the transition to fermionization is a hallmark for the inapplicability of Hubbard models.

The SIE measures plotted in Figs. 6(b) and 6(c) as a function of $\lambda$ also saturate in the transition to fermionization. The saturation of SIEs in the Mott-insulator-to-fermionization transition with two bosons in each well, however, takes place at a much larger interaction strength $(\lambda \sim 200)$ as compared to the saturation of SIEs for the superfluid-to-Mott-insulator transition $(\lambda \sim 10)$ in the case of $v=1$. Interestingly, no GOE analog for the fermionized state has been established so far and, consequently, we cannot provide a comparison of the values to which the entropies shall saturate.

The Mott insulating and the fermionized phase are, in principle, identified by the values of their entropies, as these are not yet saturated before the intrawell structure develops in the density [cf. Figs. 6(a)-6(c)]. We note that the many-body entropy measures $S_{c}, S_{n}, S_{\rho-x}$, and $S_{\rho-k}$ saturate to different numerical values for the $v=2$ and the $v=1$ cases; compare Figs. 6(b) and 6(c) and Figs. 4(b) and 4(c).

We plot the normalized one-body and two-body correlation functions $g^{(1)}$ and $g^{(2)}$ to determine how the Mott-insulatorto-fermionization transition reflects in the coherence of the many-body state; see Fig. 7. The first- and second-order coherence between different wells is similar for the fragmented and the fermionized Mott insulator, i.e., the off-diagonal parts of the normalized correlation functions $g^{(1)}$ and $g^{(2)}$ are practically identical (cf. Figs. 5 and 7). However, in contrast to the correlation functions of the fragmented Mott insulator with a single boson per site, the correlation functions for the fermionized Mott insulator feature a distinct intrawell structure (cf. Figs. 5 and 7).

\section{CONCLUSIONS}

We have shown that various information entropies can be defined to reflect and quantify many-body properties of the ground state of interacting bosons in one-dimensional lattices. These entropies serve the dual purpose to (i) identify phase transitions not seen in the single-particle properties of the system and (ii) allow a connection to known results from random matrix theory.

We demonstrated that the SIE measures are evidently in agreement with predictions for specific Gaussian orthogonal ensembles of random matrix theory for the MCTDHB results for systems with strong interactions. For sufficiently strong interactions and a single boson per site the system is fully in the Mott insulating regime and all SIE measures saturate to their respective GOE values. For sufficiently strong interactions and two bosons per site the system is fermionized and the SIE measures are saturated. For this case, however no GOE estimation is available.

All the emergent phases — the superfluid, the Mottinsulator, and the fermionized state-are identified with a distinct value of their SIE measures. Our analysis of the SIE measures is complemented by an analysis of the density and the spatial first- and second-order coherence. We showed that all emergent phases have characteristic density, correlation, and coherence patterns. We hence demonstrated that the superfluid, and the Mott-insulating and the fermionized phases of bosons in one-dimensional lattices are not only characterized uniquely by their densities and Glauber normalized correlation functions $[69,80]$, but also by their SIE values. This corroborates the fundamental connection between correlation functions and entropy measures conjectured already in Refs. [47,48].

In the transition to fermionization, intrawell structure in the densities and correlations of the many-body state is formed; this marks the breakdown of the Hubbard model. Since the SIE measures and Glauber normalized correlations allow one to identify the fermionized state, we also demonstrated two 
independent ways to assess the applicability of a Hubbard description.

Studying higher-order correlations and their "correlation holes" would be a natural extension of our work. Moreover, the crystal phase of dipolar bosons [77] and the relation of our findings for the stationary states to quench dynamics are open questions.

\section{ACKNOWLEDGMENTS}

We acknowledge insightful discussions with and comments by Norbert J. Mauser and Alex Gottlieb. Computation time at the Hazel Hen Cray XC40 cluster of the HLRS Stuttgart is gratefully acknowledged. R.R. acknowledges a UGC fellowship and B. Chakrabarti acknowledges FAPESP (Grant No. 2016/19622-0). A.G. and M.C.T. acknowledge FAPESP and A.G. thanks CNPq for financial support. B. Chatterjee acknowledges financial support from the Department of Science and Technology, Government of India, under the DST Inspire Faculty fellowship. A.U.J.L. acknowledges financial support from the Austrian Science Foundation (FWF) under Grant No. F65 (SFB "Complexity in PDEs"), and the Wiener Wissenschafts- und TechnologieFonds (WWTF) Project No MA16-066 (“SEQUEX”).
[1] I. Bloch, J. Dalibard, and W. Zwerger, Rev. Mod. Phys. 80, 885 (2008).

[2] C. Chin, R. Grimm, P. Julienne, and E. Tiesinga, Rev. Mod. Phys. 82, 1225 (2010).

[3] K. Henderson, C. Ryu, C. MacCormick, and M. G. Boshier, New J. Phys. 11, 043030 (2009).

[4] D. Jaksch, C. Bruder, J. I. Cirac, C. W. Gardiner, and P. Zoller, Phys. Rev. Lett. 81, 3108 (1998).

[5] M. Greiner, O. Mandel, T. Esslinger, T. W. Hänsch, and I. Bloch, Nature (London) 415, 39 (2002).

[6] E. Haller, R. Hart, M. J. Mark, J. G. Danzl, L. Reichsöllner, M. Gustavsson, M. Dalmonte, G. Pupillo, and H.-C. Nägerl, Nat. Lett. 466, 597 (2010).

[7] M. D. Girardeau, E. M. Wright, and J. M. Triscari, Phys. Rev. A 63, 033601 (2001).

[8] F. D. M. Haldane, Phys. Rev. Lett. 47, 1840 (1981).

[9] E. Haller, M. Gustavsson, M. J. Mark, J. G. Danzl, R. Hart, G. Pupillo, and H.-C. Nägerl, Science 325, 1224 (2009).

[10] T. Kinoshita, T. Wenger, and D. S. Weiss, Science 305, 1125 (2004).

[11] M. P. A. Fisher, P. B. Weichman, G. Grinstein, and D. S. Fisher, Phys. Rev. B 40, 546 (1989).

[12] G. E. Astrakharchik, K. V. Krutitsky, M. Lewenstein, and F. Mazzanti, Phys. Rev. A 93, 021605(R) (2016).

[13] K. Sakmann, A. I. Streltsov, O. E. Alon, and L. S. Cederbaum, Phys. Rev. Lett. 103, 220601 (2009).

[14] K. Sakmann, A. I. Streltsov, O. E. Alon, and L. S. Cederbaum, New J. Phys. 13, 043003 (2011).

[15] M. H. Beck, A. Jäckle, G. A. Worth, and H.-D. Meyer, Phys. Rep. 324, 1 (2000).

[16] I. Brouzos, S. Zöllner, and P. Schmelcher, Phys. Rev. A 81, 053613 (2010).

[17] O. E. Alon, A. I. Streltsov, and L. S. Cederbaum, Phys. Rev. A 77, 033613 (2008).

[18] A. I. Streltsov, O. E. Alon, and L. S. Cederbaum, Phys. Rev. Lett. 99, 030402 (2007).

[19] O. E. Alon, A. I. Streltsov, and L. S. Cederbaum, J. Chem. Phys. 127, 154103 (2007).

[20] J. Neuhaus-Steinmetz, S. I. Mistakidis, and P. Schmelcher, Phys. Rev. A 95, 053610 (2017).

[21] G. M. Koutentakis, S. I. Mistakidis, and P. Schmelcher, Phys. Rev. A 95, 013617 (2017).

[22] S. I. Mistakidis and P. Schmelcher, Phys. Rev. A 95, 013625 (2017).
[23] S. I. Mistakidis, T. Wulf, A. Negretti, and P. Schmelcher, J. Phys. B 48, 244004 (2015)

[24] S. I. Mistakidis, L. Cao, and P. Schmelcher, Phys. Rev. A 91 , 033611 (2015).

[25] S. I. Mistakidis, L. Cao, and P. Schmelcher, J. Phys. B 47, 225303 (2014).

[26] E. Fasshauer and A. U. J. Lode, Phys. Rev. A 93, 033635 (2016).

[27] A. U. J. Lode, Phys. Rev. A 93, 063601 (2016).

[28] A. U. J. Lode, M. C. Tsatsos, and E. Fasshauer, MCTDH-X: The Time-dependent Multiconfigurational Hartree for Indistinguishable Particles Software, http://ultracold.org (2017).

[29] A. U. J. Lode and C. Bruder, Phys. Rev. Lett. 118, 013603 (2017).

[30] O. E. Alon and L. S. Cederbaum, Phys. Rev. Lett. 95, 140402 (2005).

[31] D. M. Collins, Z. Naturforsch. a 48, 68 (1993).

[32] P. Ziesche, Int. J. Quantum Chem. 56, 363 (1995).

[33] R. O. Esquivel, A. L. Rodriguez, R. P. Sagar, M. Ho, and V. H. Smith, Phys. Rev. A 54, 259 (1996).

[34] P. Gersdorf, W. John, J. P. Perdew, and P. Ziesche, Int. J. Quantum Chem. 61, 935 (1997).

[35] P. Ziesche, O. Gunnarsson, W. John, and H. Beck, Phys. Rev. B 55, 10270 (1997).

[36] P. Ziesche, V. H. Smith Jr., M. Ho, S. Rudin, P. Gersdorf, and M. Taut, J. Chem. Phys. 110, 6135 (1999).

[37] R. Paškauskas and L. You, Phys. Rev. A 64, 042310 (2001).

[38] N. L. Guevara, R. P. Sagar, and R. O. Esquivel, J. Chem. Phys. 122, 084101 (2005).

[39] R. P. Sagar and N. L. Guevara, J. Chem. Phys. 123, 044108 (2005).

[40] K. D. Sen, J. Chem. Phys. 123, 074110 (2005).

[41] S. E. Massen and C. P. Panos, Phys. Lett. A 246, 530 (1998).

[42] S. E. Massen and C. P. Panos, Phys. Lett. A 280, 65 (2001).

[43] S. E. Massen, Ch. C. Moustakidis, and C. P. Panos, Phys. Lett. A 299, 131 (2002).

[44] M. Rigol, V. Dunjko, and M. Olshanii, Nature (London) 452, 854 (2008).

[45] L. F. Santos, F. Borgonovi, and F. M. Izrailev, Phys. Rev. Lett. 108, 094102 (2012).

[46] M. Rigol, Phys. Rev. Lett. 112, 170601 (2014).

[47] A. U. J. Lode, B. Chakrabarti, and V. K. B. Kota, Phys. Rev. A 92, 033622 (2015).

[48] I. Březinová, A. U. J. Lode, A. I. Streltsov, O. E. Alon, L. S. Cederbaum, and J. Burgdörfer, Phys. Rev. A 86, 013630 (2012).

[49] M. Olshanii, Phys. Rev. Lett. 81, 938 (1998). 
[50] A. U. J. Lode, K. Sakmann, O. E. Alon, L. S. Cederbaum, and A. I. Streltsov, Phys. Rev. A 86, 063606 (2012).

[51] A. U. J. Lode, Tunneling Dynamics in Open Ultracold Bosonic Systems, Springer Theses (Springer, Heidelberg, 2015).

[52] K. Sakmann, Many-Body Schrödinger Dynamics of BoseEinstein Condensates, Springer Theses (Springer, Heidelberg, 2011).

[53] M. C. Tsatsos and A. U. J. Lode, J. L. Temp. Phys. 181, 171 (2015).

[54] S. E. Weiner, M. C. Tsatsos, L. S. Cederbaum, and A. U. J. Lode, Sci. Rep. 7, 40122 (2017).

[55] A. U. J. Lode, A. I. Streltsov, K. Sakmann, O. E. Alon, and L. S. Cederbaum, Proc. Natl. Acad. Sci. 109, 13521 (2012).

[56] J. Grond, A. I. Streltsov, A. U. J. Lode, K. Sakmann, L. S. Cederbaum, and O. E. Alon, Phys. Rev. A 88, 023606 (2013).

[57] A. U. J. Lode, S. Klaiman, O. E. Alon, A. I. Streltsov, and L. S. Cederbaum, Phys. Rev. A 89, 053620 (2014).

[58] I. Březinová, J. Burgdörfer, A. U. J. Lode, A. I. Streltsov, L. S. Cederbaum, O. E. Alon, L. A. Collins, and B. I. Schneider, J. Phys.: Conf. Ser. 488, 012032 (2014).

[59] S. Klaiman, A. U. J. Lode, A. I. Streltsov, L. S. Cederbaum, and O. E. Alon, Phys. Rev. A 90, 043620 (2014).

[60] U. R. Fischer, A. U. J. Lode, and B. Chatterjee, Phys. Rev. A 91, 063621 (2015).

[61] A. U. J. Lode and C. Bruder, Phys. Rev. A 94, 013616 (2016).

[62] P. Molignini, L. Papariello, A. U. J. Lode, and R. Chitra, arXiv:1710.02474 (2017).

[63] A. U. J. Lode, F. S. Diorico, R. Wu, P. Molignini, L. Papariello, R. Lin, C. Léveque, L. Exl, M. C. Tsatsos, R. Chitra, and N. J. Mauser, New J. Phys. (2018), doi:10.1088/1367-2630/aabc3a.

[64] S. Dutta, M. C. Tsatsos, S. Basu, and A. U. J. Lode, arXiv:1802.02407 (2018).
[65] N. L. Guevara, R. P. Sagar, and R. O. Esquivel, J. Chem. Phys. 119, 7030 (2003).

[66] O. Penrose and L. Onsager, Phys. Rev. 104, 576 (1956).

[67] R. W. Spekkens and J. E. Sipe, Phys. Rev. A 59, 3868 (1999).

[68] E. J. Mueller, T.-L. Ho, M. Ueda, and G. Baym, Phys. Rev. A 74, 033612 (2006).

[69] R. J. Glauber, Phys. Rev. 130, 2529 (1963).

[70] S. S. Hodgman, R. G. Dall, A. G. Manning, K. G. H. Baldwin, and A. G. Truscott, Science 331, 1046 (2011).

[71] R. G. Dall, A. G. Manning, S. S. Hodgman, Wu RuGway, K. V. Kheruntsyan, and A. G. Truscott, Nat. Phys. 9, 341 (2013).

[72] T. Langen, S. Erne1, R. Geiger, B. Rauer, T. Schweigler, M. Kuhnert, W. Rohringer, I. E. Mazets, T. Gasenzer, and J. Schmiedmayer, Science 348, 207 (2015).

[73] M. C. Tsatsos, J. H. V. Nguyen, A. U. J. Lode, G. D. Telles, D. Luo, V. S. Bagnato, and R. G. Hulet, arXiv:1707.04055.

[74] T. Schweigler, V. Kasper, S. Erne, I. Mazets, B. Rauer, F. Cataldini, T. Langen, T. Gasenzer, J. Berges, and J. Schmiedmayer, Nature (London) 545, 323 (2017).

[75] A. I. Streltsov, O. E. Alon, and L. S. Cederbaum, Phys. Rev. A 81, 022124 (2010).

[76] A. I. Streltsov, K. Sakmann, O. E. Alon, and L. S. Cederbaum, Phys. Rev. A 83, 043604 (2011).

[77] B. Chatterjee and A. U. J. Lode, arXiv:1708.07409.

[78] V. K. B. Kota and R. Sahu, Phys. Rev. E 64, 016219 (2001).

[79] We repeated the calculations for $N=6, M=9$ and obtained $D_{c}=3003, D_{n}=9$ and $S_{c}=6.764$ and $S_{n}=1.925$ for the saturated entropy values. For comparison, we found $S_{c}^{\mathrm{GOE}}=$ 7.273 and $S_{n}^{\mathrm{GOE}}=2.197$.

[80] K. Sakmann, A. I. Streltsov, O. E. Alon, and L. S. Cederbaum, Phys. Rev. A 78, 023615 (2008). 